\title{
LAS DESIGUALDADES EN SALUD LABORAL Y LA INFLUENCIA DEL COVID-19 EN EL INCREMENTO DE LAS MISMAS
}

\section{Inequalities in occupational health and the influence of COVID-19 in increasing them}

\author{
Alfonso Ríos Velada* \\ Responsable de Salud Laboral, CCOO de Euskadi
}

\begin{abstract}
RESUMEN
La aparición del COVID-19 y la crisis sanitaria y económica que ha traído esta enfermedad han alterado el marco de las relaciones laborales y las características del mercado de trabajo. La necesidad de hacer frente a las problemáticas generadas ha conllevado la creación de nuevos instrumentos legales y la expansión de formas de organizar la prestación del trabajo que ya existían, como el teletrabajo. Había de forma previa en el mercado de trabajo desigualdades en el ámbito de la salud laboral debido a diversas causas. Estas desigualdades se han visto incrementadas con el COVID-19, perjudicando sobre todo a las mujeres trabajadoras en su conjunto, con especial incidencia en los riesgos psicosociales, y a todos los servicios que han sido declarados esenciales durante la pandemia, entre los cuales también hay un conjunto de sectores con mayor presencia de mujeres, por las posibilidades de contagiarse de la enfermedad.

Palabras clave: COVID-19, salud laboral, condiciones de trabajo, desigualdad.
\end{abstract}

\section{ABSTRACT}

The emergence of COVID-19 and the health and economic crisis altered the framework for labour relations and the characteristics of the labour market. The need to address the problems generated led to the creation of new legal instruments and the expansion of ways of organizing work that already existed, such as teleworking. There were previously inequalities in the labour market on occupational health due to various causes. These inequalities grew with COVID-19, especially harming working women as a whole. And they grew with special incidence on psychosocial risks, and for all the services that were declared essential in the pandemic (among which there is also a set of sectors with greater presence of women), for the risk of getting the disease.

Keywords: COVID-19, occupational health, working conditions, inequality.

\section{LABURPENA}

COVID-19ren agerpenak eta gaixotasun honek ekarritako osasun eta ekonomi krisialdiak lan-harremanen esparrua eta lan-merkatuaren ezaugarriak aldatu dituzte. Sortutako arazoei aurre egiteko beharrak lege-tresna berriak sortzea eta lehendik zeuden lana antolatzeko moduak zabaltzea, telelana, esaterako, ekarri ditu. Lan-merkatuan aldez aurretik desberdintasunak zeuden lan-osasunaren esparruan, hainbat arrazoirengatik. Desberdintasun horiek areagotu egin dira eta, batez ere, emakume langileen kalterako izan da, batez ere arrisku psikosozialetan, eta pandemian funtsezkotzat jo diren zerbitzu guztieen kalterako ere. Zerbitzu horien artean, non emakume askok lan egiten duten, gaixotasunaz kutsatzeko aukera gehiago egon dira.

Gako-hitzak: COVID-19, lan-osasuna, lan-baldintzak, desberdintasuna.

* Correspondencia a: Alfonso Ríos Velada. C/ Uribitarte 4, Bilbao (Bizkaia), España. — alfonsorios@ccoo.eus — https: //orcid. org/

Cómo citar: Ríos Velada, Alfonso. (2021). «Las desigualdades en salud laboral y la influencia del COVID-19 en el incremento de las mismas"; Lan Harremanak, 46, 144-169. (https: //doi.org/10.1387/lan-harremanak.23085).

Recibido: 28 septiembre, 2021; aceptado: 09 noviembre, 2021.

ISSN 1575-7048 - elSSN 2444-5819 / (C) 2021 UPV/EHU

(c) (i) Esta obra está bajo una licencia

Creative Commons Atribución 4.0 Internacional 


\section{Introducción}

El empleo es uno de los factores que contribuyen a la vertebración de una sociedad. De la existencia de un nivel de empleo que abarque a todas las personas que desean tenerlo, o al máximo posible de las mismas, y de que el empleo sea de calidad, se deriva otra serie de prestaciones sociales y derechos básicos para la cohesión social y una sociedad basada en la igualdad. La calidad del empleo es una pieza básica para la construcción de un orden social justo y legítimo.

La crisis sanitaria y las medidas decretadas por las autoridades para hacerle frente también han contribuido a que la sociedad haya caído en la cuenta de que existen sectores que, si bien siempre han sido necesarios y esenciales, lo han sido durante la pandemia más que nunca debido a la situación propiciada por el COVID-19. Los sectores relacionados con los cuidados, por ejemplo. El empleo de calidad en estos sectores también tiene que ver con la cohesión social. En mayor medida incluso que el empleo en otros sectores, puesto que el propio objeto del trabajo se relaciona con necesidades humanas que las personas usuarias de un servicio determinado no pueden satisfacer por sí mismas.

En la primera parte del artículo se analiza cómo la desigualdad que se da en el mundo del trabajo afecta a la salud laboral de las personas trabajadoras, creando diferencias entre las mismas en función de la exposición a distintas condiciones laborales o factores de riesgo en el trabajo. En su primera sección se analiza cómo se genera la desigualdad entre personas trabajadoras, originada por la estructuración del mundo del trabajo y las condiciones en las que éste se presta, para pasar en la segunda sección a analizar la forma en la que estas diferencias inciden sobre la desigualdad en el ámbito de la salud laboral. La tercera sección aborda una de las diferenciaciones en cuanto a la exposición a factores de riesgo que más relevancia tiene, que es la basada en la aplicación del punto de vista de género.

La segunda parte explica cómo el COVID-19 ha contribuido a agrandar las diferencias que ya existían previamente en relación a la salud laboral. En la primera sección se aborda la incidencia de la pandemia en la modificación de determinadas situaciones laborales y del mercado de trabajo en su conjunto. En la segunda sección se hace un análisis de cómo el COVID-19 ha agrandado las desigualdades en salud laboral entre las personas trabajadoras, especialmente mediante el aumento de la exposición a factores de riesgo en los servicios esenciales.

En la tercera sección, finalmente, se aplica de nuevo el punto de vista de género en el ámbito de la salud laboral, puesto que han sido las mujeres trabajadoras las más perjudicadas por estos factores de riesgo impuestos por el COVID-19, fundamentalmente por la expansión del teletrabajo. 


\section{Población trabajadora y desigualdad asociada al trabajo}

\subsection{Desigualdad en el mercado de trabajo}

La desigualdad que existe en los mercados de trabajo de los diferentes países en relación a las condiciones laborales es una característica de los mismos. Son varias las diferenciaciones que atraviesan esos mercados de trabajo, siendo las de género o edad algunas de las más conocidas. Las diversas formas de contratación que posibilita llevar a cabo la legislación espańola, contemplándose la temporalidad y el empleo a tiempo parcial, así como las desigualdades salariales, son causa de la expansión de la precariedad laboral que afecta a diversos colectivos de personas trabajadoras.

El discurso empresarial tradicional ha basado la necesidad de flexibilizar estas diversas formas de contratación, que segmentan el mercado de trabajo y crean colectivos de personas trabajadoras que tienen más o menos derechos y prestaciones, en la adaptabilidad de las empresas para hacer frente a los diversos cambios de la demanda de bienes y servicios. Lo dejaba claro Jon Bilbao ya en 1999 en el ciclo de conferencias que organizó la Escuela Universitaria de Relaciones Laborales de la Universidad del País Vasco, las cuales quedaron recogidas en el segundo número de la revista Lan Harremanak. Según el entonces director del departamento de relaciones laborales de la patronal vasca Confebask, los cambios sólo pueden ser respondidos mediante adaptación y flexibilidad, ya que «de manera rígida, uniforme, no se puede hacer frente al cambio» (Bilbao et al., 2000: 218).

Las empresas y representantes patronales han identificado estas rigideces como imposibilidades para ajustar plantillas mediante despidos cuando las necesidades lo requieran. También como imposibilidades para contratar de forma temporal durante los periodos en los que la demanda lo exija. El modelo defendido desde las patronales conlleva un aumento de la desigualdad al generar colectivos de personas desempleadas, pero también hace aumentar la desigualdad entre las personas que tienen un empleo, puesto que coexisten personas contratadas de forma indefinida y otras contratadas de forma temporal. Esta desigualdad tiene su origen en el proceso de fragmentación del colectivo de personas trabajadoras, que existe desde el mismo inicio de la relación laboral al darse diferentes condiciones de inserción en la empresa.

Posteriormente se introduce el término de empleabilidad, como conjunto de medidas que posibilitan el acceso a nuevos empleos tras los despidos, lo que implica la rotación de personas trabajadoras por diversas empresas. Esto beneficia a las empresas, puesto que es parte del sistema que permite contratar y despedir con facilidad, y fomenta que «el desgaste más rápido de la fuerza de trabajo se subsana por el hecho de que se crea la posibilidad de una sustitución rápida de la misma» (Baylos, 2003: 58). Las facilidades para despedir a personal fijo y 
reemplazarlo, mediante personal habitualmente contratado de forma temporal, acaba por imponer un rendimiento de trabajo más intenso, lo que implica a su vez aumentos de productividad. Esta intensificación de la producción, que se ejecuta mediante la contratación temporal y las personas trabajadoras de «quita y pon», es una de las explicaciones al hecho de que, a pesar de encontrarnos en una época de progreso tecnológico que tendría que mejorar las condiciones laborales, se produzcan empeoramientos en los indicadores que miden la carga física en los puestos de trabajo (Santos, 2008: 23).

Pese al intento de implantación de esa empleabilidad, el propio empleo, que es mecanismo de integración social y fuente de ingresos para la satisfacción de necesidades, "cada vez presenta un acceso más restringido y no supone una fuente de seguridad suficiente para todas las personas que acceden al mismo» (Martín et al., 1997: 63). Desempleo y precariedad laboral son causa directa de pobreza y exclusión social. Es habitual que existan mercados de trabajo que no pueden prevenir una tasa estructural de desempleo que coexiste con la precariedad laboral de las personas ocupadas, colectivo este último al que tampoco se le garantiza un salario digno que permita eludir situaciones de pobreza (Babiano et al., 2018: 52).

Las diferencias salariales son una fuente de desigualdad constante en el mercado de trabajo, y contribuyen a la diferenciación de colectivos de personas trabajadoras. Hay relación entre el nivel salarial, medido como ganancia económica que aporta el trabajo, y las diferentes formas de contratación que se dan en el mercado de trabajo. Las personas contratadas de forma temporal son las que más alternan periodos de empleo con periodos en los que no se trabaja, recibiendo en estos últimos prestaciones de desempleo inferiores al salario que proporcionaría el trabajo remunerado. Asimismo, las personas contratadas a tiempo parcial reciben la remuneración salarial que les corresponde según el porcentaje de jornada por el que hayan sido contratadas, con el perjuicio que esto implica en comparación con las personas contratadas a tiempo completo.

Dentro de la población trabajadora, las personas más jóvenes son las grandes perjudicadas por el deterioro de las condiciones laborales, por las formas más precarias de contratación y por los bajos salarios. El estudio elaborado por Cantó (2019: 73) afirma que, si en los países del sur de Europa la pobreza afecta a una menor proporción de jóvenes que en los países del norte, es porque en los países del sur se retrasa la emancipación del hogar parental. Por ello, en estos países los principales mecanismos de mitigación de la pobreza no serían ni el acceso al mercado de trabajo ni la protección social, sino el "colchón familiar» que presta ayuda. No es sólo el hecho de no poder acceder a un salario digno proporcionado por un empleo de calidad, sino que además este hecho no permite dar continuidad ni estabilidad a un sistema de cotización, requisito para el acceso a otras prestaciones, como la de desempleo o la de jubilación. 
El fenómeno denominado pobreza salarial, entendido como la situación en la que contar con un puesto de trabajo que proporcione un salario no sea suficiente para salir de situaciones de pobreza, no es algo nuevo. A mediados de los ańos 90 del pasado siglo xx eran estos mismos países del sur de Europa los que registraban los índices de pobreza global y de pobreza salarial más altos. En el conjunto de los países que en aquel momento componían la Unión Europea, el 10\% de la población vivía en "hogares asalariados pobres» (Mendialdea et al., 2005: 59). En el conjunto del estado, por su parte, la crisis económica que se inicia en el año 2008 influye en el aumento de esta pobreza salarial. Según el estudio elaborado por Martínez et al. (2018: 201), la tasa de riesgo de pobreza en la población ocupada aumentó de un $11,7 \%$ en el año 2008 a un 14,8\% en 2015.

Los efectos que las diversas crisis económicas tienen sobre el empleo son innegables. Si bien es cierto que en la crisis económica abierta en 2020 a causa de la pandemia del COVID-19 la utilización de los expedientes de regulación temporal de empleo (ERTE) ha contribuido al mantenimiento de la relación laboral para un gran número de personas, en otros casos las empresas han recurrido a expedientes de regulación de empleo (ERE) que han significado un incremento del número de despidos.

El modelo productivo español lleva aparejado un mercado de trabajo con protagonismo de sectores cuyas características de empleo de mano de obra se basan en la facilidad del reemplazo de la misma. Los ejemplos más característicos serían la construcción o la hostelería, sectores en los que prolifera un modelo de contratación temporal, y en los cuales las personas trabajadoras tienen, en la mayoría de los casos, baja cualificación. El empleo en estos sectores, en los que la precariedad laboral era algo característico antes de la aparición del COVID-19, ha sido especialmente castigado por los efectos colaterales de la pandemia (Álvarez, 2020: 162).

Una vez analizadas las causas de la desigualdad en el mercado de trabajo y en qué forma se manifiesta esta, es necesario explicar las repercusiones que se trasladan al ámbito de la salud laboral. Existen modelos de gestión, como la subcontratación, que profundizan también en la generación de desigualdades en este espacio.

\subsection{Desigualdad en relación a la salud laboral}

El mercado de trabajo es un espacio plural en lo relativo a la forma en la que las condiciones de trabajo pueden afectar a la salud. Forma de contratación, estabilidad laboral o trabajar en ocupaciones que puedan ser más o menos nocivas, peligrosas o insalubres, generan desigualdad entre las personas trabajadoras en relación a la exposición a factores que puedan propiciar un accidente de trabajo, una enfermedad profesional o patologías derivadas de los riesgos psicosociales. 
Los diversos puestos de trabajo que hay en una empresa pueden ser influidos por diversos vectores que hacen que el deterioro de la salud debido al trabajo no sea el mismo para unas personas que para otras, lo que también influye en la percepción de la defensa de la salud laboral que sea necesario llevar a cabo. La ocupación que cada persona desarrolla en la empresa, por ejemplo, tomando la diferenciación más clásica y sencilla de visualizar, como puede ser personas trabajadoras de planta o taller por un lado y de oficina por el otro, nos permite ver que son las condiciones de trabajo a las que cada una de esas personas se ve sometida las que influyen de forma más decisiva en esto.

Hay situaciones reales que ilustran esta diferente percepción, como es la de los numerosos casos de silicosis ${ }^{1}$ que comenzaron a aparecer en el sector de marmolería hace quince años en la CAPV y que no dejan de producirse. Afectan en mayor medida a personal de taller que a personal de oficina, por estar más expuesto a polvo de sílice el primer colectivo que el segundo. Fueron las personas que trabajaban en taller en estas empresas las primeras en exigir evaluaciones de riesgos higiénicos y medidas preventivas, así como en denunciar los incumplimientos legales de las empresas para que éstas pagasen indemnizaciones de daños y perjuicios a quien hubiese contraído silicosis. En ocasiones, el personal de oficina percibía estas acciones como una amenaza para su propia estabilidad laboral y para la continuidad de la empresa, ya que estaba expuesto en menor medida al polvo de sílice ${ }^{2}$, desarrollándose la enfermedad en muchos menos casos.

El ordenamiento jurídico en materia de seguridad y salud en el trabajo tiene un precepto legal que establece de forma clara el objetivo de atenuar las diferencias entre personas trabajadoras, que es el artículo 15.1.h) de la Ley 31/1995, de 8 de noviembre, de prevención de Riesgos Laborales ${ }^{3}$. Establece que la empresa debe adoptar medidas que antepongan la protección colectiva antes que la indi-

\footnotetext{
${ }^{1}$ La silicosis, reconocida como enfermedad profesional para diversos sectores productivos en los cuales hay presencia de polvo de sílice, ha experimentado un aumento de su incidencia no sólo en la CAPV, sino en el conjunto del estado. Para ahondar en el incremento de la incidencia es recomendable la lectura del artículo «La remergencia de la silicosis como enfermedad profesional en España, 1990-2019». El artículo ha sido escrito por Alfredo Menéndez-Navarro, Catherine Cavalin, Montserrat García-Gómez y Alin Gherasim, y forma parte del número 95 de la Revista Espańola de Salud Pública, publicado en 2021. Está disponible en el siguiente enlace: https://www.mscbs. gob.es/biblioPublic/publicaciones/recursos_propios/resp/revista_cdrom/VOL95/ORIGINALES/ RS95C_202108106.pdf

2 El polvo de sílice libre no sólo ocasiona silicosis, sino que también puede ocasionar cáncer. Está reconocido como agente cancerígeno por el Real Decreto 257/2018, de 8 de mayo, por el que se modifica el Real Decreto 1299/2006, de 10 de noviembre, por el que se aprueba el cuadro de enfermedades profesionales en el sistema de la Seguridad Social y se establecen criterios para su notificación y registro (BOE núm.109, de 5 de mayo de 2018). Disponible para su análisis en este enlace: https://www.boe.es/buscar/doc.php?id=BOE-A-2018-6046.

${ }^{3}$ El texto de la Ley 31/1995, de 8 de noviembre, de prevención de Riesgos Laborales (BOE núm. 269, de 10 de noviembre de 1995) se puede consultar en este enlace: https://www.boe.es/buscar/act.php?id=BOE-A-1995-24292.
} 
vidual. Es obligatorio para la empresa implantar de forma prioritaria y en primer lugar medidas que protejan a todo el colectivo de personas trabajadoras expuestas a un riesgo. Posteriormente, y sólo como complemento a esta protección colectiva, se implantarán medidas destinadas a proteger a las personas trabajadoras de forma individual.

En el caso de un taller de marmolería con exposición a polvo de sílice, por ejemplo, la empresa tiene que implantar en primer lugar mecanismos de extracción del polvo en el taller o en la maquinaria en la que se registren emisiones, y donde este sistema no resulte suficiente, tendrá que dotar a las personas trabajadoras de equipos de protección individual para las vías respiratorias. Si sólo proporcionase estos equipos de protección individual sin implantar sistemas de extracción, se estaría incumpliendo el citado artículo 15.1.h) de la Ley de prevención de Riesgos Laborales.

Por otro lado, poder hacer un descanso en la jornada laboral es considerado incluso un factor que previene la aparición de dolencias de tipo psicosocial asociadas a la carga de trabajo, tanto física como mental. Esta posibilidad no está al alcance de todas las personas trabajadoras, influyendo en la misma la forma de contratación, puesto que «los trabajadores con contrato temporal tienen menos libertad para tomarse un descanso en el trabajo (26'5\%) en comparación con los trabajadores con contrato permanente (39\%)» (Benach et al., 2004). Aliviar la tensión provocada por la carga de trabajo mediante un descanso, lo que a su vez también disminuye la posibilidad de tener un accidente laboral, está más al alcance de las personas que tengan un contrato fijo y es más difícil para quienes tengan un contrato temporal. De esta manera, la temporalidad en la contratación influye en la siniestralidad laboral, y propicia escenarios como el desarrollado entre los ańos 2012 y 2018, años entre los que «el incremento registrado en el índice de incidencia de la siniestralidad laboral en los contratos temporales $(28,1 \%)$ casi quintuplica el producido en los indefinidos (6,4\%)»(Chulvi, 2019).

La fragmentación de un colectivo de personas trabajadoras que desempeñan su tarea en un mismo centro de trabajo se da también en ocasiones a través de la subcontratación de obras y servicios. Personas que están en un mismo proceso productivo y en un mismo centro de trabajo tienen condiciones laborales distintas, también en el ámbito de la prevención de riesgos laborales. Para prestar una obra o servicio determinado a una empresa principal, las empresas que aspiran a ser subcontratadas compiten entre sí por hacer sus ofertas más atractivas. Para ello, estas empresas realizan recortes que empeoran las condiciones de trabajo de sus plantillas y que incluso en ocasiones conllevan «suprimir, retirar o no implantar medidas preventivas encaminadas a que no se generen daños a la salud de las personas trabajadoras» (Ríos, 2020: 225). La supresión de medidas preventivas tiene consecuencias en forma de accidentes de trabajo o enfermedades profesionales. 
La subcontratación de obras y servicios y la externalización de tareas es ya una característica fundamental del mercado de trabajo. El actual discurso empresarial dominante, basado en la demanda de flexibilidad para la búsqueda de la competitividad y la adaptación permanente al mercado, conlleva la fragmentación del proceso productivo de una empresa en partes, cuyas operaciones a su vez las realizan las plantillas de empresas subcontratadas. Estas empresas pueden ir cambiando y ser sustituidas unas por otras, por lo que la pugna entre ellas para mantener el contrato que permite realizar una obra o servicio, con la consiguiente degradación de condiciones de trabajo, es constante.

Las demandas que modifican las condiciones de trabajo no proceden de la propia empresa, sino de la empresa principal, que es quien tutela y dirige todo el proceso. Hay una alteración de la organización del trabajo que es externa, y esto tiene también consecuencias sobre los riesgos psicosociales a los que las personas trabajadoras de las empresas subcontratadas, o que realizan actividades que han sido externalizadas, están expuestas.

Si hay una operación que anteriormente era realizada por las propias empresas, pero que está siendo subcontratada y externalizada cada vez en mayor medida, es la que aglutina los procesos de creación, aplicación y mantenimiento de herramientas y programas informáticos. Las personas trabajadoras de las empresas que se encargan de estas herramientas precisan instrucciones muy concretas sobre los requisitos de la empresa principal o empresa "cliente», y en ocasiones la información ni es todo lo concreta que debería ni llega a tiempo. También es frecuente que esos requisitos de la empresa principal conlleven alteraciones e irregularidades en horarios de trabajo y calendarios laborales, con ausencia de carga de trabajo o necesidad de realizar horas extras según el momento.

Estos requisitos se derivan de que una empresa principal en cualquier momento «introduzca cambios en la planificación, o modifique los requisitos o funcionalidades exigidas, provocando problemas organizativos y de gestión» (Castillo, 2007: 110) como demanda para las empresas externalizadas que llevan a cabo las tareas asociadas a las herramientas informáticas, demanda a la que estas empresas tienen que prestar una atención constante. La dificultad que conlleva la atención a estas demandas se traslada a la plantilla en forma de obstáculos para la organización de la vida personal y la conciliación con el ámbito doméstico y de los cuidados, siendo causa de incremento del riesgo psicosocial asociado al trabajo.

En determinados espacios se articulan mecanismos que pretenden equiparar en derechos la protección de la salud frente a los riesgos psicosociales de las personas trabajadoras de empresas principales y de las empresas que para esta operen como subcontrata. La Dirección del Organismo Estatal de la Inspección de Trabajo y Seguridad Social aborda este punto en el Criterio Técnico 104/2021, sobre actuaciones de la Inspección de Trabajo y Seguridad Social en riesgos psi- 
cosociales $^{4}$. En el punto 1.3 de este documento, donde se aborda la aplicación de las medidas preventivas, hay un apartado específico sobre coordinación de actividades empresariales en el que se establece que cuando haya varias empresas presentes en un centro de trabajo han de ponerse en marcha los mecanismos de coordinación para la implantación de esas medidas. El punto 1.7 del mismo documento, por su parte, establece que el incumplimiento de este deber de coordinación puede ser motivo de adopción de medidas de requerimiento y, en su caso, de inicio del procedimiento sancionador.

También en la jurisprudencia se producen avances en materia de salud laboral para los sectores y empresas cuyas actividades características son subcontratadas en un mayor número de ocasiones. Uno de los últimos ejemplos es la STS $725 / 2020^{5}$, que reconoce el síndrome del túnel carpiano como enfermedad profesional para las camareras de piso de los hoteles. Este colectivo de trabajadoras, muy habitualmente subcontratadas, soporta ritmos de trabajo intensos que conllevan exposición a riesgos musculoesqueléticos que se derivan de los movimientos repetitivos y las posturas forzadas. La sentencia referida establece que puede darse la calificación de enfermedad profesional para otras profesiones o actividades más allá de las contenidas en los listados de cada epígrafe del Anexo I del Real Decreto 1299/2006, de 10 de noviembre, por el que se aprueba el cuadro de enfermedades profesionales en el sistema de la Seguridad Social y se establecen criterios para su notificación y registro ${ }^{6}$, y que lo relevante es el contenido de las tareas y la exposición a las condiciones de trabajo que provoquen la dolencia.

Hay diferencias en la salud laboral entre las personas trabajadoras, motivadas por las diversas condiciones de trabajo a las que se ven expuestas, y también existen instrumentos mitigadores de esas diferencias. Sobre las diferencias, uno de los ámbitos que es imprescindible analizar de forma específica es el de la desigualdad entre hombres y mujeres.

\subsection{Salud laboral y punto de vista de género}

Existen diferencias en relación a cómo algunas condiciones de trabajo afec$\tan$ a las mujeres y a los hombres, y esto ha de tenerse en cuenta a la hora de

${ }^{4}$ El texto del Criterio Técnico 104/2021, sobre actuaciones de la Inspección de Trabajo y Seguridad Social en riesgos psicosociales, se puede consultar en la página web del Ministerio de Trabajo y Economía Social. Está disponible en el siguiente enlace: https://www.mites.gob.es/itss/ITSS/ITSS_ Descargas/Atencion_ciudadano/Criterios_tecnicos/CT_104_21.pdf

5 La sentencia STS 725/2020, de 11 de febrero de 2020 (ECLI: ES:TS:2020:725) puede consultarse en este enlace: https://adriantodoli.com/wp-content/uploads/2020/05/STS_725_2020.pdf

${ }^{6}$ El citado Anexo I y el Real Decreto 1299/2006, de 10 de noviembre, por el que se aprueba el cuadro de enfermedades profesionales en el sistema de la Seguridad Social y se establecen criterios para su notificación y registro (BOE núm. 302, de 19 de diciembre de 2006), pueden ser consultados en este enlace: https://www.boe.es/buscar/act.php?id=BOE-A-2006-22169 
abordar la prevención de riesgos laborales. De entre las condiciones de trabajo que pueden generar un daño a la salud, aquellas en las que más claramente se produce esta diferenciación de género serían las que son susceptibles de influir sobre la salud psicosocial.

Los riesgos psicosociales que se dan en el trabajo acaban en ocasiones afectando a la salud mental, y la dificultad de su correcta evaluación deriva, entre otras cosas, de la falta de homogeneidad sobre los efectos que causan en las personas, porque la misma intensidad de un mismo riesgo puede afectar en mayor medida a una persona que a otra.

La falta de autonomía en el trabajo es un ejemplo de riesgo psicosocial. En la actualidad hay en nuestro mercado de trabajo más mujeres en puestos de ejecución que en puestos directivos, lo que implica que el conjunto de las mujeres trabajadoras dispone de menos autonomía y se ve sometido a un mayor control en sus puestos de trabajo que el conjunto de los hombres trabajadores. Esta distribución conlleva también que el desarrollo profesional del conjunto de las mujeres trabajadoras, en caso de poder ser llevado a cabo, va a comenzar desde posiciones inferiores que el del conjunto de los hombres trabajadores.

También la doble presencia y la exigencia de conciliación de la vida laboral y familiar afecta en mayor medida a las mujeres que a los hombres. En el ámbito del trabajo, por un lado, y en el ámbito que abarca el espacio doméstico y el espacio de los cuidados, por otro lado, se producen exigencias que afectan al conjunto de las personas trabajadoras, teniendo las mujeres que ocuparse del segundo de los ámbitos en más ocasiones que los hombres. Debido a esto, el riesgo para la salud psicosocial relacionado con la doble presencia, que deriva de que se produzcan exigencias que son simultáneas en el tiempo y que conllevan además en ocasiones delimitaciones temporales muy estrictas, afecta en mayor medida a las mujeres.

En este escenario influyen otros elementos que incrementan este riesgo psicosocial para las mujeres trabajadoras. «Las tareas domésticas típicamente femeninas siguen siendo aquellas que tienen unos condicionantes temporales y espaciales muy rígidos» (Rodríguez et al., 2017: 183), por lo que existe mayor dificultad para compaginarlas con los requerimientos del puesto de trabajo, siendo por ello tareas más difícilmente programables. Por el contrario, en el ámbito doméstico los hombres realizan tareas de rango temporal menos restringido. Esta falta de equiparación redunda en un incremento del riesgo psicosocial asociado a la doble presencia para las mujeres trabajadoras.

Las mujeres trabajadoras están también afectadas por la brecha salarial. Cobran menos que los hombres por realizar un mismo trabajo, lo cual implica no sólo un agravio comparativo, sino también una falta de valoración del trabajo realizado por las mujeres. Esta ausencia de valoración justa de lo que alguien 
hace en su trabajo contribuye a hacer que se pierda el sentido del mismo para la persona que lo realiza, generando un nuevo riesgo psicosocial. Un estudio elaborado con datos del año 2018 por el sindicato CCOO (2021: 19) establece que las mujeres ganan al año en el conjunto del estado 5.726 euros menos que los hombres, y que sería necesario incrementar en un $27 \%$ el salario medio de las mujeres para equipararlo al de los hombres.

Para ese mismo año 2018, en la CAPV, la Encuesta de Estructura Salarial que elabora el Instituto Nacional de Estadística establece que el salario medio de los hombres fue de 31.970,30 euros, mientras que el de las mujeres fue de 24.757,18 euros. La diferencia entre unos y otras es de 7.213,12 euros, también en perjuicio para las mujeres. En la CAPV esta desigualdad salarial no se puede explicar argumentando que el nivel de formación de las mujeres es inferior. El estudio elaborado por Larrañaga et al. (2017: 216) sobre las desigualdades de género asociadas al mundo del trabajo establece que el $55 \%$ de las mujeres tiene estudios superiores, mientras que en el colectivo de los hombres el porcentaje es del $49 \%$.

Más allá de los aspectos psicosociales, hay otros riesgos que es necesario contemplar desde el punto de vista de género, puesto que también afectan de forma distinta a mujeres y a hombres. En relación a la exposición profesional a contaminantes químicos, todas las sustancias liposolubles pueden acumularse con mayor facilidad en personas con más proporción de tejido graso. Según los datos aportados por Valls-Llobet, el colectivo femenino puede llegar a presentar un $15 \%$ más de materia grasa que el colectivo masculino, "lo que hace que las mujeres sean posibles bioacumuladores químicos de los productos» (Valls-Llobet, 2010: 152) a los que se encuentran expuestas, bien a través de la contaminación ambiental o de las exposiciones que se dan en los puestos de trabajo.

La segregación ocupacional horizontal del mercado de trabajo hace que las mujeres estén más expuestas a riesgos que generan enfermedades profesionales que a riesgos que generan accidentes de trabajo. Por poner ejemplos de sectores profesionales concretos, en los relacionados con los cuidados, como serían las residencias de la tercera edad o el sector de ayuda a domicilio, de presencia mayoritaria femenina, hay múltiple exposición a riesgos musculoesqueléticos derivados de posturas forzadas, movimientos repetitivos o manipulación manual de cargas, susceptibles de generar dolencias como el síndrome del túnel carpiano, patología típica de estos sectores.

Por el contrario, los accidentes laborales ocasionados por caídas a distinta altura, golpes o atrapamientos, son característicos de sectores como la construcción o la industria, donde la presencia masculina es mayoritaria. La cultura preventiva arraigada en los centros de trabajo ha estado centrada en prevenir el accidente de trabajo, como hecho inmediatamente perceptible, frente a la enfermedad profesional, que implica un periodo de latencia temporal que 
hace que la misma no se perciba en el momento de la exposición al factor susceptible de provocarla. Esto ha hecho que las mujeres trabajadoras hayan sido las grandes olvidadas de la cultura preventiva, debido a que están en «trabajos generalmente menos expuestos a riesgos de muerte o accidente muy grave» (González, 2011: 108), y ello pese a que su deterioro de salud originado por las condiciones de trabajo lo producen las enfermedades profesionales, pero a más largo plazo.

Cuando exista sospecha de que hay un caso de enfermedad profesional de las reconocidas como tales en el ya mencionado Real Decreto 1299/2006, por el que se aprueba el cuadro de enfermedades profesionales, tanto el personal facultativo del sistema público de salud como el del servicio de prevención correspondiente que realiza la vigilancia de la salud en el trabajo a cada persona trabajadora, tienen que comunicarlo al organismo competente en materia de seguridad y salud en el trabajo de cada comunidad autónoma (en la CAPV, a Osalan, el Instituto Vasco de Seguridad y Salud Laborales), que a su vez lo comunicará al Instituto Nacional de la Seguridad Social y a la mutua correspondiente para su correcta calificación como enfermedad profesional. Esta obligación de comunicación se encuentra en el artículo 5 del propio RD 1299/2006.

Lo más habitual es que se realicen comunicaciones de sospecha de origen profesional de hipoacusias derivadas del ruido en el trabajo, antes que dolencias de tipo musculoesquelético. Según los datos publicados por Osalan, el Instituto Vasco de Seguridad y Salud Laborales, en el año 2019 se produjeron 436 comunicaciones de sospecha de enfermedad profesional en la CAPV. De todas estas, «la hipoacusia fue con diferencia el diagnóstico más frecuente también en el año 2019 con 108 comunicaciones» (Idiazabal et al., 2021: 19).

Esto conlleva que, al ser el ruido una característica del trabajo en sectores masculinizados, como la industria y la construcción, no siendo una característica tan habitual en sectores con gran presencia de mujeres trabajadoras, como los cuidados o la limpieza, el colectivo de hombres trabajadores se ve favorecido a la hora de que se comuniquen sus dolencias para el reconocimiento del origen profesional. Hay un sesgo de género en la comunicación de sospecha de enfermedades profesionales.

La jurisprudencia corrige este sesgo en ocasiones, como lo ha hecho la sentencia N.o 250/2020 del Juzgado de lo Social N. 2 de Bilbao, que ha reconocido el origen profesional de la sordera de una trabajadora del sector de colectividades, cuyo puesto de trabajo está en el comedor escolar de un centro educativo. La sentencia considera probado que la trabajadora desarrolla su tarea sometida a un nivel diario equivalente de ruido superior a 80 decibelios, nivel que se alcanza de forma habitual en sectores industriales, y que en un comedor escolar deriva del eco generado por los escolares usuarios del comedor, de la maquinaria de lavado de platos y cubiertos o de los choques y golpes producidos 
entre útiles de menaje. Se reconoce por tanto el origen profesional de una dolencia característica de sectores masculinizados en un sector feminizado, lo que genera un precedente para otras mujeres trabajadoras del sector de colectividades que presenten hipoacusia derivada del ruido en el trabajo.

Las variables de desigualdad analizadas, que afectan al ámbito de la salud laboral, han sufrido modificaciones. La irrupción de la crisis económica y sanitaria que ha generado el COVID-19 desde el año 2020 ha significado un ensanchamiento de estas brechas de desigualdad. No sólo en el ámbito de la salud laboral, sino también en el conjunto del mercado de trabajo.

\section{Incremento de la desigualdad en salud laboral por influencia del COVID-19}

\subsection{Influencia del COVID-19 en las relaciones laborales y el mercado de trabajo}

La inestabilidad económica que ha venido aparejada a la crisis sanitaria del COVID-19 ha socavado los cimientos del mercado de trabajo y ha afectado a las condiciones de trabajo y vida de las personas. El primer requisito para que las relaciones laborales y el puesto de trabajo no generen riesgos psicosociales es que no exista incertidumbre. En los primeros momentos de la pandemia, en determinadas ocasiones no se sabía si había que ir a trabajar o no ni quién debía de hacerlo en cada centro de trabajo. Era también fuente de incertidumbre cuál iba a ser el futuro de la empresa donde se trabajaba, así como si se iba a estar afectado por un ERTE o ir al desempleo. Según Navarro (2020: 49) durante los dos primeros meses de la pandemia 3,4 millones de personas se vieron afectadas por ERTEs, mientras que, en un solo mes, concretamente el de abril de 2020, el paro registrado creció en casi 300.000 personas, cifra que nunca se había alcanzado antes en el plazo de un mes.

$\mathrm{Al}$ introducirse condicionalidad y vulnerabilidad en la situación laboral, son también otros ámbitos los que se debilitan, puesto que la incertidumbre se extiende a los gastos referentes a hipoteca o alquiler, así como a los generados por otras actividades indispensables (Benach et al., 2020). Esta ausencia de certezas afecta a la salud psicosocial de las personas.

En relación a la posibilidad de contagios y propagación del virus, el trabajo y el desplazamiento al mismo en transporte público han sido vectores de transmisión, debido a las interacciones con personas y objetos. Son espacios en los que "una simple utilización de reglas de higiene destinadas a establecer barreras espaciales no basta» (Voguel, 2020). En este contexto surgía una nueva incertidumbre que afectaba al mundo del trabajo en relación a «si la exposición 
al COVID-19 se debía considerar un riesgo grave e inminente, muy ligada tal cuestión a si este era un riesgo laboral o no" (López, 2020: 213).

Durante los primeros meses de la pandemia también se generó una situación que se convirtió en fuente de incertidumbre para las personas trabajadoras que estaban de incapacidad transitoria (IT) por accidente de trabajo. Las mutuas correspondientes emitían partes de alta sin haber llevado a cabo una nueva revisión del estado de salud de las personas afectadas, y alegaban que las mismas ya estaban recuperadas y listas para incorporarse al puesto de trabajo. La persona a quien se emitía este parte de alta cuenta con diez días hábiles para impugnar el mismo ante el Instituto Nacional de la Seguridad Social, según el artículo 4.2 del Real Decreto 1430/2009, de 11 de septiembre, por el que se desarrolla reglamentariamente la Ley 40/2007, de 4 de diciembre, de medidas en materia de Seguridad Social, en relación con la prestación de incapacidad temporal ${ }^{7}$.

No obstante, las oficinas del INSS permanecían cerradas debido a la situación sanitaria, lo que en un primer momento hacía imposible la impugnación. Desde la propia Seguridad Social se habilitaron aplicaciones informáticas para llevar a cabo estas tramitaciones, pero, aparte de que no todo el conjunto de la población trabajadora puede tener acceso a este tipo de herramientas, las mismas fallaban repetidamente debido a problemas técnicos. Como se ve, la situación vivida por personas en situación de IT a las cuales la mutua dio el alta médica no fue sólo de incertidumbre, sino que también generó indefensión.

Por otra parte, las personas que están en la economía sumergida o el empleo informal también han sufrido en lo relativo a su subsistencia los efectos de la situación generada por el COVID-19. Según los datos recabados por Macías (2020: 116), el empleo informal constituye el 90\% del total del empleo en los países en desarrollo, el 67\% en los países emergentes y el 18\% en los países desarrollados. Este grupo de personas trabajadoras perdió su fuente directa de ingresos en las épocas de confinamiento, no habiendo generado previamente ninguna posibilidad de cobertura por prestación económica alguna debido a la falta de cotización.

Una vez analizados los principales problemas que el COVID-19 ha traído al conjunto de las personas trabajadoras y los factores de inestabilidad que ha generado en el mercado de trabajo, es necesario prestar una especial atención a la repercusión en los servicios esenciales. También a las soluciones dadas al hecho de que algunas personas tuviesen que acudir a su puesto de trabajo de forma obligatoria mientras la mayoría estaba confinada en su domicilio.

7 El Real Decreto 1430/2009, de 11 de septiembre, por el que se desarrolla reglamentariamente la Ley 40/2007, de 4 de diciembre, de medidas en materia de Seguridad Social, en relación con la prestación de incapacidad temporal (BOE núm. 235, de 29 de septiembre de 2009), puede consultarse en este enlace: https://www.boe.es/buscar/doc.php?id=BOE-A-2009-15442. 


\subsection{Influencia del COVID-19 en la salud laboral del personal de los servicios esenciales}

La crisis sanitaria originada por el COVID-19 ha puesto en valor el trabajo de determinados sectores profesionales, algunos de los cuales hasta el momento habían pasado desapercibidos para una gran parte de la sociedad. Todos los sectores relacionados con el ámbito de los cuidados a personas enfermas o dependientes, la limpieza, la atención sanitaria, la recogida y tratamiento de residuos, el personal que trabaja en supermercados, el mantenimiento y la reparación de averías urgentes, etc., fueron decretados como esenciales ${ }^{8}$ desde el primer momento de la pandemia.

El trabajo en estos sectores implicaba exposición al riesgo de contraer el COVID-19 en todo momento, incluso para las personas trabajadoras que acudían a su puesto en transporte público en un momento en que no era posible mantener distancia de seguridad en el mismo, no había garantías de que esa distancia de seguridad fuese efectiva frente al riesgo de contagio ni había disponibilidad de equipos de protección individual. Asimismo, la naturaleza de las tareas de estos sectores hacía imposible la implantación del trabajo a distancia, una de las medidas que desde el primer momento se destacaron como las que era necesario intentar implantar para frenar la expansión del COVID-199. La percepción de poder sufrir un contagio al tener que acudir al puesto de trabajo ha sido también fuente de riesgo psicosocial para estas personas.

Entre quienes tuvieron que acudir a su puesto de trabajo durante esa primera época de la pandemia también había diferencias, marcadas por el nivel salarial y la necesidad de tener que ir a trabajar, aún con síntomas de COVID-19, o no verse en la necesidad de tener que acudir al puesto de trabajo. El estudio elaborado por el Instituto Sindical de Trabajo, Ambiente y Salud y la Universitat Autònoma de Barcelona sobre las condiciones de trabajo durante los primeros meses de pandemia concluye que el porcentaje de las personas que acudieron a su puesto de trabajo con síntomas de COVID-19 «es casi el doble entre aquellas trabajadoras y trabajadores a quienes habitualmente su salario no les permite cubrir las necesidades básicas del hogar» (Salas-Nicás et al., 2020: 33), si lo comparamos con aque-

${ }^{8}$ El anexo que se incluyó en el Real Decreto-ley 10/2020, de 29 de marzo, por el que se regula un permiso retribuido recuperable para las personas trabajadoras por cuenta ajena que no presten servicios esenciales, con el objetivo de reducir la movilidad de la población en el contexto de la lucha contra el COVID-19 (BOE núm. 87, de 29 de marzo de 2020), enumeraba los sectores a cuyas personas trabajadoras no les sería de aplicación el citado permiso retribuido recuperable, por ser precisamente personas trabajadoras de los denominados servicios esenciales. El texto legal en su totalidad puede consultarse en este enlace: https://www.boe.es/buscar/doc.php?id=BOE-A-2020-4166.

9 El artículo 5 del Real Decreto-ley 8/2020, de 17 de marzo, de medidas urgentes extraordinarias para hacer frente al impacto económico y social del COVID-19 (BOE núm. 73, de 18 de marzo de 2020), establecía el carácter preferente del trabajo a distancia. El texto legal en su totalidad puede consultarse en este enlace: https://www.boe.es/buscar/act.php?id=BOE-A-2020-3824. 
llas otras trabajadoras y trabajadores cuyo salario les permite cubrir esas necesidades. Según los datos que ofrece el propio estudio, en el primer caso fue un 18,2\% de las personas trabajadoras, mientras que en el segundo caso fue un $10,5 \%$.

Dentro de los servicios esenciales es necesario hacer una especial mención a los sectores relacionados con los cuidados, en los que hay gran presencia de mujeres trabajadoras. En el ámbito de los cuidados «la precarización de las condiciones de trabajo de estos colectivos feminizados ya era una realidad y la pandemia ha agravado la situación» (Llorens et al., 2021). Los sectores relacionados con los cuidados prestan servicios indispensables, lo que tendría que significar un incentivo para que fuesen sectores de titularidad pública. No obstante, se han ido produciendo recortes y limitaciones en el gasto público. Debido a esto, por un lado, se ha producido una transferencia de este trabajo de cuidados a las familias, "pero las transferencias no se están produciendo sólo del sector público a los hogares sino también al mercado» (Larrañaga, 2014: 34), lo que implica un mayor protagonismo de la iniciativa privada.

La entrada de la iniciativa privada en el sector de los cuidados significa en muchas ocasiones un aumento de la precariedad laboral y un deterioro de las condiciones de trabajo. La necesidad de atenciones y cuidados va a seguir existiendo, y no afrontar esa tarea directamente desde los servicios públicos, favoreciendo que empresas privadas la gestionen, implica que se deja «en manos del mercado esta responsabilidad y el resultado es la creación de puestos de trabajo infrapagados» (Santos, 2008: 28).

Pese a este proceso de degradación de condiciones de trabajo, en el marco de la crisis generada por el COVID-19 estos sectores han demostrado más que nunca ser fundamentales para el funcionamiento de la sociedad. No en vano, que las familias puedan «externalizar» los cuidados a las personas que trabajan en ese ámbito, hace que los miembros de esas familias puedan acudir a sus respectivos puestos de trabajo.

Como se ha indicado, el trabajo a distancia fue una de las medidas prioritarias para la limitación del contacto social que implican la permanencia en el centro de trabajo y el desplazamiento al mismo en transporte público. A esta medida no pueden acogerse ni las personas que trabajan en los cuidados ni las personas que trabajan en el sector sanitario. Asimismo, entre las personas que trabajan en el sector industrial se dio en los primeros momentos de la pandemia una situación de desigualdad en relación a esto, al coexistir en una misma empresa personal técnico o administrativo que, por la naturaleza de su actividad, pudo acogerse al trabajo a distancia, con personal de taller o planta que tenía que acudir al centro de trabajo al encontrarse allí la maquinaria que tenía que utilizar. Muchas empresas industriales fueron parte de los servicios esenciales debido a que los bienes que producían eran necesarios para hacer frente a la extensión del COVID-19 o para el tratamiento de personas contagiadas. 
Al hecho de no poder acogerse al trabajo a distancia se sumaba la imposibilidad de recurrir a la autoridad laboral por riesgo grave e inminente en caso de que no existiesen medidas preventivas para frenar la expansión del virus y hacer frente a posibles contagios, aspecto abordado en el apartado anterior. Esta figura, regulada en el artículo 21 de la Ley 31/1995, de prevención de Riesgos Laborales, implica que las personas trabajadoras, bien de forma directa o bien a través de sus representantes, pueden paralizar un puesto de trabajo o una serie de tareas del mismo, con interrupción de la actividad e incluso abandono del centro de trabajo, cuando consideren que la actividad en cuestión entrañe riesgo grave e inminente para la salud o la vida. En este precepto legal se dice de forma expresa que la paralización habrá de comunicarse de forma inmediata a la empresa y a la autoridad laboral, ratificando o anulando esta última la paralización acordada en el plazo de veinticuatro horas. Es la Inspección de Trabajo quien de forma habitual realiza la visita al emplazamiento concreto, para que la autoridad laboral decida en un sentido o en otro.

El criterio técnico N. ${ }^{\circ} 103 / 2020$, del Organismo Estatal de la Inspección de Trabajo y Seguridad Social ${ }^{10}$, sobre actuaciones de la Inspección de Trabajo y Seguridad Social relativas a la habilitación contenida en el Real Decretoley 21/2020, de 9 de junio, en relación a las medidas de prevención e higiene para hacer frente a la crisis sanitaria ocasionada por el COVID-19 en los centros de trabajo, enumeraba una serie de actuaciones tales como la vigilancia del cumplimiento de las medidas preventivas e higiénicas establecidas por la autoridad sanitaria, la realización de requerimientos y la extensión de actas de infracción. No se menciona, y queda por tanto excluida, la ratificación de los supuestos de paralización de actividad ante riesgo grave e inminente.

La consideración del COVID-19 no como un riesgo laboral, sino como un riesgo de salud pública, aunque presente en los centros de trabajo, permitía que las situaciones de riesgo por falta de medidas preventivas e higiénicas fuesen comunicadas por la Inspección de Trabajo a la autoridad sanitaria mediante remisión de informes. El tercer punto del anexo de este criterio técnico N. ${ }^{\circ} 103 / 2020$ sí establecía que la autoridad sanitaria podía adoptar medidas como el cierre preventivo de centros de trabajo y la suspensión de actividades. Que la paralización de actividades por riesgo grave e inminente, mecanismo legal cuyo objetivo es proteger la salud y la vida de las personas trabajadoras, no pudiese ser ratificada de forma directa por la Inspección de Trabajo retrasa la citada paralización, lo

10 El criterio 103/2020 del Organismo Estatal de la Inspección de Trabajo y Seguridad Social, sobre actuaciones de la Inspección de Trabajo y Seguridad Social relativas a la habilitación contenida en el Real Decreto-ley 21/2020, de 9 de junio, en relación con las medidas de prevención e higiene para hacer frente a la crisis sanitaria ocasionada por la Covid-19 en los centros de trabajo, se puede consultar en la página web del Ministerio de Trabajo y Economía Social. Está disponible en el siguiente enlace: https://www.mites.gob.es/itss/ITSS/ITSS_Descargas/Atencion_ciudadano/Criterios_ tecnicos/CT_103_2020.pdf. 
cual hace que el mecanismo no resulte útil cuando el objetivo era frenar la propagación del virus.

El Organismo Estatal de la Inspección de Trabajo y Seguridad Social clarificó aún más estas actuaciones mediante una instrucción ${ }^{11}$ en cuyo punto primero se afirmaba que «no procede la utilización por los funcionarios de la Inspección de Trabajo y Seguridad Social de la facultad de paralizar trabajos, tareas o actividades por riesgo grave e inminente, si se apreciase la existencia de riesgo de exposición y de contagio por incumplimiento de las medidas preventivas acordadas por el Ministerio de Sanidad». En el punto tercero de la misma instrucción se establecía que tampoco procederá la paralización de trabajos y tareas en centros, servicios y establecimientos sanitarios determinados como tales por el Ministerio de Sanidad, ni en centros sociales de personas mayores, dependientes o con discapacidad.

El reconocimiento de los contagios de COVID-19 como dolencias de origen profesional también ha sido diferente para personas trabajadoras que compartían espacios de trabajo, como ha ocurrido en los centros de trabajo del sector sanitario. El Real Decreto-ley 28/2020, de 22 de septiembre, de trabajo a distancia ${ }^{12}$, incluyó en su disposición adicional cuarta que los contagios serían considerados accidente de trabajo para las personas que trabajasen directamente en los sectores sanitario y socio-sanitario, por exponerse al riesgo en el ejercicio de la profesión y en la prestación del servicio.

Queda reconocido el COVID-19 como contingencia profesional para, por ejemplo, el personal médico y de enfermería de un hospital y para los gerocultores y gerocultoras de una residencia de la tercera edad, pero no para el personal de una subcontrata que se dedica a la limpieza en cualquiera de esos dos centros de trabajo. Siendo este último colectivo también parte de los servicios esenciales, no va a tener acceso directo a beneficiarse de las mismas prestaciones económicas, que son mayores en los casos de contingencia profesional que en los de contingencia común.

Posteriormente, el Real Decreto-ley 3/2021, de 2 de febrero, por el que se adoptan medidas para la reducción de la brecha de género y otras medidas en los ámbitos de la Seguridad Social y económicos ${ }^{13}$, estableció en su artículo 6

${ }^{11} \mathrm{La}$ instrucción referida puede consultarse en este enlace: https://www.cea.es/wp-content/ uploads/2020/04/DOC-03-Informe-ITSS.pdf.

12 El Real Decreto-ley 28/2020, de 22 de septiembre, de trabajo a distancia (BOE núm.253, de 23 de septiembre de 2020), puede consultarse en este enlace: https://www.boe.es/buscar/act. php?id=BOE-A-2020-11043.

13 El Real Decreto-ley 3/2021, de 2 de febrero, por el que se adoptan medidas para la reducción de la brecha de género y otras materias en los ámbitos de la Seguridad Social y económicos (BOE núm. 29, de 3 de febrero de 2021), puede consultarse en este enlace: https://www.boe.es/buscar/act. php?id=BOE-A-2021-1529. 
que los contagios por COVID-19 para ese mismo personal sanitario o socio-sanitario habrían de ser reconocidos como enfermedad profesional y no como accidente de trabajo, pero nuevamente sin incluir al personal de las subcontratas que realizase sus tareas en esos centros de trabajo y no fuese personal sanitario o socio-sanitario. Esta consideración existirá mientras persistan las medidas preventivas decretadas por las autoridades para hacer frente a la crisis sanitaria ocasionada por el COVID-19.

Finalmente, el Procedimiento de actuación para los servicios de prevención de riesgos laborales frente a la exposición al SARS-COV-2 aprobado por el Ministerio de Sanidad, Consumo y Bienestar Social ${ }^{14}$ cita ambas referencias legales en su apartado 8.1 para que sean correctamente aplicadas en los sistemas de notificación que han de llevar a cabo los servicios de prevención.

Las personas que trabajaban en los servicios esenciales, obligadas a acudir a su puesto de trabajo durante la pandemia, estuvieron en el escenario descrito. Pero las personas que permanecieron confinadas en su domicilio y pudieron acogerse al teletrabajo también se han visto expuestas a otros factores de riesgo, especialmente las mujeres.

\subsection{El teletrabajo y su influencia en los riesgos psicosociales de las mujeres trabajadoras}

Entre los múltiples cambios que la pandemia del COVID-19 ha traído a las relaciones laborales y los espacios donde éstas se dan, destaca la implantación del teletrabajo en un gran número de empresas. Si bien esta forma de organizar la prestación del trabajo ya existía, la necesidad de reducir las interacciones sociales, entre ellas las que se daban en el centro de trabajo de forma presencial y en el transporte público para acudir al mismo, ha contribuido de forma importante a su extensión, afectando en mayor medida a las mujeres trabajadoras, ya que «en el contexto del COVID-19 fueron más las mujeres (34,7\%) las que teletrabajaron que los hombres (26,6\%)» (López, 2021).

Esta nueva situación se ha dado en un escenario que era previo a la situación de crisis sanitaria y que ha sido explicado en el apartado 2.3, escenario en el cual

14 El Procedimiento de actuación para los servicios de prevención de riesgos laborales frente a la exposición al SARS-COV-2 fue uno de los primeros que el Ministerio de Sanidad, Consumo y Bienestar Social difundió, con el objetivo de dar a conocer las medidas adecuadas para contener la extensión del COVID-19 que pudiese darse a través de las interacciones entre personas en los centros de trabajo. Desde el inicio de la pandemia hasta la actualidad ha tenido diversas versiones que modificaban puntos concretos del mismo, siempre que hubiese nuevas directrices a trasladar e implementar, derivadas del conocimiento del virus y de sus formas de transmisión. La última versión del procedimiento, de fecha 16 de julio de 2021, está disponible en la web del Ministerio de Sanidad, Consumo y Bienestar Social en el siguiente enlace: https://www.mscbs.gob.es/profesionales/saludPublica/ ccayes/alertasActual/nCov/documentos/Proteccion_Trabajadores_SARS-CoV-2.pdf. 
las mujeres trabajadoras se ven afectadas en mayor medida que los hombres por el riesgo psicosocial derivado de la doble presencia. No en vano, antes de la pandemia del COVID-19 eran también más las mujeres que se acogían a situaciones de reducción de jornada para el cuidado de hijas e hijos o familiares dependientes.

El teletrabajo contribuye por ello a incrementar este riesgo psicosocial para las mujeres en mayor medida que para los hombres. Las mujeres han estado teletrabajando más que los hombres desde el domicilio en una época en la cual los centros escolares han estado cerrados por efecto del COVID-19. La situación que se generó fue grave, y esto se demuestra por el hecho de que, desde el primer momento del cierre de los centros educativos, el foco principal de la problemática originada por este cierre no estaba «en cómo afectaba negativamente a las necesidades educativas y de sociabilidad de niños, adolescentes y jóvenes, sino en el impacto que tenía en la conciliación» (Coscubiela, 2021: 167). La incompatibilidad existente para compaginar empleo y atención a hijos e hijas la asume en parte el sistema educativo sin que sea su función. En ausencia del sistema educativo, la tarea del cuidado se traslada a las familias «a tiempo completo», recayendo de forma prioritaria sobre las mujeres.

En esta fase, en la que las hijas e hijos en edad escolar tuvieron que permanecer en el domicilio, pero progresivamente iba posibilitándose la reincorporación de las personas que estaban teletrabajando al centro de trabajo de forma presencial, «las mujeres se quedaron más teletrabajando en casa y los hombres tendían más a volver al centro de trabajo» (CCOO de Madrid, 2020: 96). Esta situación ha propiciado que las mujeres hayan tenido que simultanear, en mayor medida que los hombres, la prestación laboral con el cuidado de hijos e hijas, por lo que la problemática de la doble presencia ha experimentado una nueva forma de afectar a las mujeres, dándose en el mismo tiempo y espacio y teniendo que atenderse a la vez. La solución aplicada por las mujeres que teletrabajaban ha sido la prolongación de la jornada laboral.

La nueva forma en la que la doble presencia afecta a las mujeres trabajadoras ha traído también el desarrollo de nuevos instrumentos legales encaminados a la implantación de medidas preventivas, como son algunos de los artículos incluidos en el ya referido Real Decreto-ley 28/2020, de trabajo a distancia. El artículo 15 de este texto legal establece el derecho a la protección en materia de seguridad y salud en el trabajo, de conformidad con lo establecido en la Ley 31/1995, de prevención de Riesgos Laborales, y su normativa de desarrollo, para las personas en situación de teletrabajo, lo que en la práctica las equipara a las personas trabajadoras que desarrollen la actividad de manera presencial en el centro de trabajo. El artículo 16, por su parte, aborda de forma específica la evaluación de riesgos y la planificación de la actividad preventiva, «poniendo especial atención en los factores psicosociales, ergonómicos y organizativos», y es- 
tableciendo que han de ser especialmente contemplados en la evaluación y de cara a la implantación de medidas preventivas «la distribución de la jornada, los tiempos de disponibilidad y la garantía de los descansos y desconexiones durante la jornada».

El hecho de que las mujeres se vean más a menudo en situación de teletrabajo implica para ellas una sobrecarga en la responsabilidad «dado que la conciliación se convierte en un problema que se les atribuye directamente a ellas" (Gálvez, 2020: 3). Por ello, que mujeres y hombres no se acojan de forma equitativa al teletrabajo contribuye a que el mercado laboral se convierta a su vez en un mecanismo más de reproducción de los roles de género. Si a esta situación se añade, como se ha explicado, que el sistema educativo tuvo que ser paralizado de forma total por efecto de la crisis sanitaria derivada del COVID-19, el escenario resultante conlleva que «la imposibilidad de conciliar el empleo remunerado y el trabajo de cuidados se haga aún más patente» (González et al., 2020: 43).

Además de todo esto, la supuesta ventaja de que la propia persona trabajadora se organice para la prestación de la tarea a través del teletrabajo, no es tal. La prestación de la tarea puede acabar diluyéndose en el tiempo hasta abarcar espacios a lo largo de las veinticuatro horas del día, precisamente por tener que prestar atención a los cuidados en horario laboral. Esto ocurre con mayor frecuencia a las mujeres porque han compaginado en mayor medida la situación de teletrabajo con los cuidados. Por ello, el teletrabajo no puede ser considerado una herramienta que favorezca la conciliación de la vida laboral, familiar y personal. Antes, bien al contrario, en una situación en la que las mujeres asumen en mayor medida que los hombres tareas domésticas y relacionadas con los cuidados, distintas brechas de género asociadas al teletrabajo "pueden terminar replicando las desigualdades y segregaciones propias del mercado de trabajo tradicional» (López et al., 2021: 74).

No es la única manera en la cual la expansión del teletrabajo va a afectar a la salud psicosocial de las mujeres trabajadoras. Si las mujeres han teletrabajado más que los hombres en la situación generada por el COVID-19, esto quiere decir asimismo que los hombres han permanecido en mayor medida de forma presencial en los centros de trabajo. Por ello, se establece una diferenciación en las perspectivas de desarrollo de la carrera profesional entre ambos colectivos, puesto que existen más posibilidades de promoción si se está de forma presencial en la empresa que si se trabaja desde casa. Esto se traduce también en un incremento del riesgo psicosocial para las mujeres trabajadoras, puesto que, si la situación derivada de la extensión del teletrabajo va a facilitar que los hombres tengan aún más posibilidades de promoción, continuarán ocupando los puestos de mayor responsabilidad como hasta ahora e incluso en mayor medida. El resultado final es la disminución de la autonomía y de la capacidad de decisión del conjunto de las mujeres trabajadoras en comparación con el conjunto de los hombres. 
Si bien esta situación de menor autonomía del conjunto de las mujeres trabajadoras ya se estaba dando, la implantación en muchas empresas del teletrabajo que se ha derivado de la situación del COVID-19 va a contribuir a su extensión y perpetuación. Las personas que ocupan puestos de más responsabilidad en las organizaciones "casi nunca se acogen al teletrabajo, con lo que implícitamente se transmite el mensaje de que para prosperar en la organización siguen primando los criterios de presencialidad" (Pérez et al., 2009: 75).

\section{Conclusiones}

El COVID-19 ha modificado diversos espacios de nuestra sociedad. La incidencia en el mercado de trabajo es innegable, siendo uno de los cambios fundamentales la exposición a nuevos factores de riesgo que han influido en la salud de la clase trabajadora y en cómo las condiciones de trabajo han alterado la misma. La exposición a condiciones de trabajo distintas ya existía debido, entre otras causas, a la necesidad de adaptación que ante las fluctuaciones del mercado las empresas exigen a sus plantillas. Este factor de riesgo, generador de desigualdad en el conjunto del mercado de trabajo, ya repercutía en la salud laboral de las personas, afectando de forma distinta según la ocupación.

En una misma empresa es la realización de las diversas tareas de cada una de las personas la que establece su exposición a condiciones de trabajo distintas, derivándose de este hecho la desigualdad en el ámbito de la salud laboral. Las diferentes exposiciones abarcan, entre otros factores, seguridad en el trabajo, entendida como la posibilidad de tener un accidente laboral, presencia de contaminantes químicos en el ambiente de trabajo o riesgos psicosociales, por nombrar sólo los más claros e identificables. La subcontratación de obras o servicios por parte de una empresa principal a otra u otras incide también en que las personas trabajadoras de estas últimas tengan más riesgo de sufrir un daño a la salud derivado del trabajo.

Las mujeres trabajadoras, por su parte, salen peor paradas que el colectivo de hombres trabajadores en relación al deterioro de la salud motivado por el trabajo. La doble presencia, que afecta en mayor medida a aquellas que a estos, y que implica la asimilación de las obligaciones del puesto de trabajo y la necesidad de atender igualmente el ámbito doméstico y de los cuidados, es causa de riesgo psicosocial.

Si bien este era el escenario previo a la pandemia, la aparición de la misma ha alterado la organización del trabajo en las empresas, puesto que estas han sido espacios en los cuales existía la posibilidad de contraer el COVID-19, lo que ha- 
cía imprescindible la implantación en las mismas de las medidas decretadas por las autoridades sanitarias. El mercado de trabajo en su conjunto también se ha visto alterado por la situación sanitaria y las medidas para evitar la propagación del virus.

Quienes más han padecido la nueva situación son las personas trabajadoras de los servicios esenciales. Su trabajo es imprescindible, en unas ocasiones debido a su relación con la mitigación de los efectos de la situación sanitaria, o en otras ocasiones debido a su relación con necesidades humanas que han de atenderse, como las derivadas de los cuidados o de diversos tipos de abastecimiento. Estas personas tuvieron que estar en su puesto de trabajo mientras una gran parte de la población permanecía confinada. La posibilidad de contraer el COVID-19 ha sido mayor para estas personas de los servicios esenciales. Su situación ha evidenciado la relación existente entre la salud pública y la salud laboral.

El confinamiento domiciliario fue, especialmente en los primeros momentos de la pandemia, una medida fundamental de entre las adoptadas por las autoridades sanitarias para evitar la propagación del virus. La necesidad por parte de las empresas de buscar fórmulas que permitiesen el mantenimiento de la actividad productiva, o al menos de una parte de ella, ha motivado una expansión del teletrabajo. Se ha realizado en su totalidad desde el domicilio de la persona trabajadora, lo que ha originado un incremento de los factores de riesgo psicosocial, que han perjudicado más a las mujeres debido al fenómeno de la doble presencia, que de forma previa les afectaba ya en mayor medida que a los hombres.

\section{Bibliografía}

Álvarez, Henar (2020). «El futuro del trabajo después de la pandemia y los empleos del futuro", Gaceta Sindical, 35, 155-174. Disponible en: https://www.ccoo.es/272578cd 60bb40ce42f9818b348f23e6000001.pdf (accedido: 21-09-2021).

BABIANO, José y TÉBAR, Javier (2018). «Clase, precariedad y ejército global de reserva en el capitalismo flexible. Algunas consideraciones en perspectiva histórica», Anuario IET de Trabajo y Relaciones Laborales, 5, 43-58. Disponible en: https://ddd.uab.cat/pub/ anuarioiet/anuarioiet_a2018v5/anuarioiet_a2018v5p43.pdf (accedido: 21-09-2021).

Baylos, Antonio (2003). Las relaciones laborales en España 1978-2003, Madrid: Ediciones GPS.

Benach, Joan y Amable, Marcelo (2004). «Desigualdades en salud laboral», El País, Disponible en: https://elpais.com/diario/2004/11/02/salud/1099350007_850215.html (accedido: 21-09-2021).

BenACH, Joan, Alòs, Ramón y JóDAR, Pere (2020). «La nueva normalidad no puede ser una nueva precariedad», CTXT, Disponible en: https:/www.upf.edu/es/web/focus/opinio/-/ asset_publisher/lk4diAT4aI2f/content/id/234930169/maximized\#.YUBgTLgzaUl (accedido: 21-09-2021). 
Bilbao, Jon y Serrano, Felipe (2000). «El futuro de las relaciones laborales», Lan Harremanak, 2, 211-235. Disponible en: https:/ojs.ehu.eus/index.php/Lan_Harremanak/ article/view/6684 (accedido: 21-09-2021).

Cantó SÁnchez, Olga (2019). "Desigualdad, redistribución y políticas públicas: ¿Hay una brecha generacional?», Información Comercial Española, 908, 65-79. Disponible en: http:// www.revistasice.com/index.php/ICE/article/view/6826/6871 (accedido: 22-09-2021).

Castillo, Juan José (2007). El trabajo fluido en la sociedad de la información. Madrid: Miño y Dávila Editores.

CCOO (2021). Cuentas claras para acabar con la brecha salarial. Madrid: Confederación sindical de CCOO. Disponible en: https://www.ccoo.es/d6358833d528a8efd7ef9ec0c12e8fc3000001.pdf (accedido: 22-09-2021).

CCOO DE MADrid (2020). Salud laboral \& género: especial atención a la COVID-19 y su impacto en las mujeres, Madrid: Ediciones GPS.

Chulvi, Berta (2019). "Accidentes: la huella más dramática de la precariedad», Por Experiencia, 81. Disponible en: https://porexperiencia.com/condiciones-de-trabajo/accidentes-la-huella-mas-dramatica-de-la-precariedad (accedido: 28-10-2021).

Coscubiela, Joan (2021). La pandemia del capitalismo. Barcelona: Ediciones Península.

GÁlvez Mozo, Ana (2020). «Mujeres y teletrabajo: más allá de la conciliación de la vida laboral y personal», Oikonomics. Revista de economia, empresa y sociedad, 13, 1-9. Disponible en: https://oikonomics.uoc.edu/divulgacio/oikonomics/_recursos/ documents/13/agalvez_Oikonomics13_esp.pdf (accedido: 22-09-2021).

González Celis, Inés y González Llama, Amaia (2020). «Donde se sostiene la vida: cuidados, cuerpo(s) y comunidad en tiempos de pandemia», Inguruak, 69, 39-56. Disponible en: https://inguruak.eus/index.php/inguruak/article/view/69-2020art03/113 (accedido: 22-09-2021).

González Gómez, M. ${ }^{a}$ Fernanda (2011). «Salud laboral y género. Apuntes para la incorporación de la perspectiva de género en el ámbito de la prevención de riesgos laborales», Medicina y Seguridad del Trabajo, 57-supl. 1, 89-114: Disponible en: https:// pdfs.semanticscholar.org/05fd/4d7fb90a76fa16942bc67f3f829b88f978fe.pdf (accedido: 22-09-2021).

Idiazabal Garmendia, Jasone y López Echaniz, Idoia (2021). Comunicaciones de sospechas de enfermedad profesional en la Comunidad Autónoma de Euskadi 2019, OsalanInstituto Vasco de Seguridad y Salud Laborales. Disponible en: https://www.osalan. euskadi.eus/contenidos/informacion/informes_anuales_siniestralida/es_def/adjuntos/ informe_sep_2019.pdf (accedido: 29-10-2021).

Larrañaga Sarriegi, Mertxe (2014). "Tiempos de precariedad y desamparo», Viento Sur, $134,32-41$.

Larrañaga Sarriegi, Mertxe y Martínez Tola, Elena (2017). «Desigualdades económicas de género en la Comunidad Autónoma del País Vasco", Ekonomiaz, 91, 209-247. Disponible en: https://www.euskadi.eus/web01-a2reveko/es/k86aEkonomiazWar/ ekonomiaz/abrirArticulo?idpubl=87\&registro=14 (accedido: 22-09-2021).

Llorens, Clara y López, Montse (2021). «Llueve sobre mojado: mujeres en trabajos invisibilizados durante la pandemia", Por Experiencia, 85. Disponible en: https://porexperiencia.com/condiciones-de-trabajo/llueve-sobre-mojado-mujeres-en-trabajos-invisibilizados-durante-la-pandemia (accedido: 22-09-2021).

López Bermúdez, Montse (2021). «Teletrabajo con perspectiva de género y salud laboral», Por Experiencia, 85. Disponible en: https://porexperiencia.com/dossier/teletrabajo-con-perspectiva-de-genero-y-salud-laboral (accedido: 22-09-2021). 
López Fernández, Rubén (2020). «Los servicios de prevención de riesgos laborales en tiempos del coronavirus", Lan Harremanak, 43, 202-215. Disponible en: https://doi. org/10.1387/lan-harremanak.21714 (accedido: 22-09-2021).

López Igual, Purificación y Rodríguez Modroño, Paula (2021). «Factores de desigualdad entre teletrabajadores en Europa», Revista de Economía Crítica, 31, 62-78. Disponible en: http://revistaeconomiacritica.org/sites/default/files/2-PurificacionLopezIgual-PaulaRodriguezModronyo_Factores-de-desigualdad-entre-teletrabajadores.pdf (accedido: 22-09-2021).

Macías, Iñigo (2020). "Pandemia y desigualdad en España: cuando llueve sobre mojado», Gaceta Sindical, 35, 113-120. Disponible en: https://www.ccoo.es/272578cd60bb40c e42f9818b348f23e6000001.pdf (accedido: 21-09-2021).

Martín, Idoia y Simón, Lola (1997) «De la sociedad del trabajo a la sociedad de la exclusión", Inguruak, 17, 61-74.

Martínez Martín, Rafael, García Moreno, José Manuel y Lozano Martín, Antonio Manuel (2018). «Trabajadores pobres en España. El contexto de la crisis económica como marco para comprender la desigualdad», Papeles de Población, 98, 185-218. Disponible en: https://rppoblacion.uaemex.mx/article/view/8880/9525 (accedido: 26-10-2021).

Mendialdea, Bibiana y Álvarez, Nacho (2005). «Ajuste neoliberal y pobreza salarial: los working poor en la Unión Europea», Viento Sur, 82, 56-64.

Navarro, Vicenc (2020) Pandemia, economía y estado del bienestar (versión autoeditada) Disponible en: http://www.vnavarro.org/wp-content/uploads/2020/10/Pandemiaeconom\%C3\%ADa-y-Estado-del-Bienestar.-Vicen\%C3\%A7-Navarro.pdf (accedido: 22-09-2021).

Pérez Sánchez, Carmen y Gálvez Mozo, Ana María (2009). «Teletrabajo y vida cotidiana: Ventajas y dificultades para la conciliación de la vida laboral, personal y familiar», Athenea Digital, 15, 57-79. Disponible en: https:/www.redalyc.org/articulo. oa?id=53712929004 (accedido: 27-10-2021).

Ríos Velada, Alfonso (2020). «La negociación colectiva como instrumento para frenar los daños a la salud laboral derivados de la precariedad», Lan Harremanak, 44, 218 237. Disponible en: https://doi.org/10.1387/lan-harremanak.22213 (accedido: 2209-2021).

Rodríguez Álvarez, Arantxa y Ugidos Olazabal, Arantza (2017). «Tiempos, trabajos y desigualdad de género en la Comunidad Autónoma del País Vasco y el Estado español», Ekonomiaz, 91, 165-207. Disponible en: https://www.researchgate.net/publication/324476415_Tiempos_trabajos_y_desigualdad_de_genero_ en_la_Comunidad_Autonoma_del_Pais_Vasco_y_el_Estado_espanol_Time_ work_and_gender_inequality_in_the_Basque_Country_and_Spain (accedido: 22-09-2021).

Salas-Nicás, Sergio, Llorens-Serrano, Clara, Navarro, Albert y Moncada, Salvador (2020). Condiciones de trabajo, inseguridad y salud en el contexto del COVID-19. Barcelona. UAB, ISTAS-CCOO. Disponible en: https://istas.net/sites/default/ files/2021-01/InformeCOTS_01072020-1_1.pdf (accedido: 24-09-2021).

Santos Ortega, Antonio (2008). «Las relaciones laborales en los márgenes del mercado de trabajo", Arxius de Ciències Socials, 18, 25-37. Disponible en: https://roderic.uv.es/ bitstream/handle/10550/19440/SANTOS.pdf?sequence $=1$ \&isAllowed=y (accedido: 24-09-2021). 
Valls-Llobet, Carme (2010). «Contaminación ambiental y salud en las mujeres», Investigaciones feministas, 1, 149-159. Disponible en: https://www.researchgate.net/ publication/277819305_Contaminacion_ambiental_y_salud_de_las_mujeres (accedido: 24-09-2021).

Voguel, Laurent (2020). «El trabajo, ángulo ciego en la crisis de Covid-19», Viento Sur, Disponible en: https://vientosur.info/el-trabajo-angulo-ciego-en-la-crisis-de-covid-19/ (accedido: 22-09-2021). 